\title{
Control of Carbon Flux to Acetate Excretion During Growth of Escherichia coli in Batch and Continuous Cultures
}

\author{
By E. M. T. EL-MANSI AND W. H. HOLMS* \\ Department of Biochemistry, University of Glasgow, Glasgow G12 8QQ, UK
}

(Received 13 April 1989; revised 26 June 1989; accepted 24 July 1989)

\begin{abstract}
During growth of Escherichia coli ML308 on pyruvate in a continuous culture (turbidostat) or batch culture, flux of carbon into the cells exceeds the amphibolic capacity of the central pathways. This is balanced by diversion of carbon flux to acetate excretion which in turn diminishes the efficiency of carbon conversion to biomass [g] dry wt (mol substrate) $)^{-1}$ ]. However, restriction of carbon supply in a chemostat diminishes flux to acetate excretion and at a dilution rate $(D=\mu)$ of $0.35 \mathrm{~h}^{-1}$ or less, no flux to acetate excretion was sustained thus permitting perfect balance between carbon input on the one hand, and the output to biosynthesis and energy generation on the other. This, in turn, improves the efficiency of carbon conversion to biomass. Inclusion of 3-bromopyruvate (an inhibitor of pyruvate dehydrogenase) at a concentration which diminishes growth rate $(\mu)$ to $0.35 \mathrm{~h}^{-1}$ or less also prevented flux to acetate excretion. Furthermore, in a family of fluoroacetate-resistant strains, excessive flux of pyruvate was balanced by diversion of carbon flux to lactate excretion rather than acetate and a higher growth rate $\left(\mu=0.63 \mathrm{~h}^{-1}\right)$ was sustained.
\end{abstract}

\section{INTRODUCTION}

Growth of a micro-organism requires the biosynthesis of a specific range of monomers (Morowitz, 1968) most of which are assembled into polymers (Dawes \& Large, 1982; Neidhardt et al., 1987) which form the bulk of the new biomass (Holms, 1987). Aerobically, Escherichia coli can grow on a wide range of compounds as sole source of carbon (Holms, 1986). Each different carbon source is taken into the cell and fed into the central metabolic pathways which contain the enzymes required to interconvert some 30 intermediates, rather more than half of which are phosphorylated; the remainder are carboxylic acids. Some of these intermediates (eight or nine) serve as precursors for the biosynthesis of a wide range of monomers which, in turn, are the raw material for the biosynthesis of polymers (Neidhardt et al., 1987). Many of the uptake and anabolic processes require the input of energy in various forms (NADPH, ATP, PEP, acetyl$\mathrm{CoA}$, transmembrane proton gradient etc.) and under aerobic conditions these are generated by the flow of carbon and its oxidation through the central pathways. The high-energy forms thus generated are converted to their low-energy forms by the anabolic routes and recycled to the central pathways. It follows that the outputs of the central pathways are water, $\mathrm{CO}_{2}$, energy and biosynthetic precursors. The inputs are carbon source, oxygen and some inorganic ions. Under carbon limitation (in a chemostat), the flux of carbon into the cell (input) matches fluxes to outputs for biosynthesis and energy generation. However, when carbon flux exceeds the metabolic capacity of the central pathways, the system is balanced in one of three ways.

1. Intermediates in excess of requirements can be converted to storage polymers, e.g. glycogen (Dawes \& Senior, 1973; Dietzler et al., 1979).

Abbreviations: PDH, pyruvate dehydrogenase; PEP, phosphoenolpyruvate. 


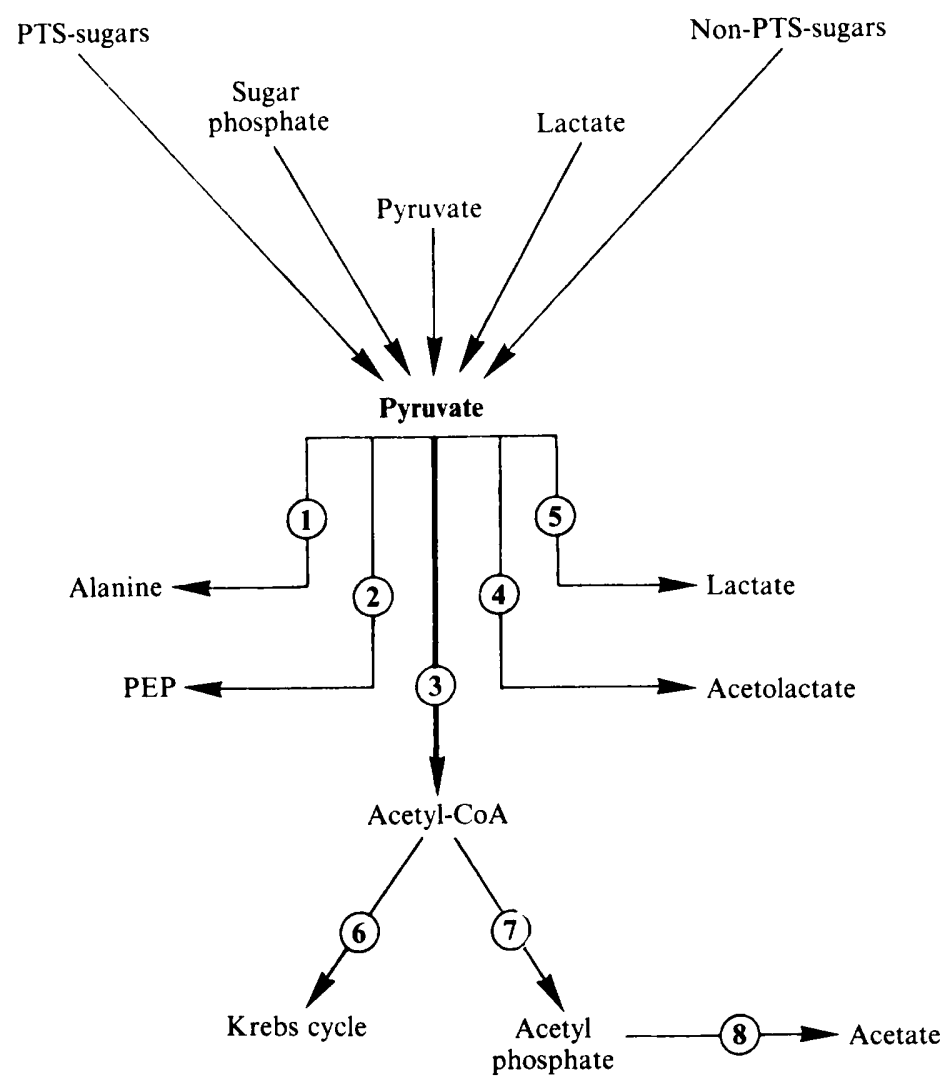

Fig. 1. Convergence of various metabolic routes on pyruvate on the route to acetate excretion in $E$. coli. Enzymes are as follows: 1, glutamate:pyruvate transaminase; 2, PEP synthase; 3 , pyruvate dehydrogenase; 4 , acetolactate synthetase; 5 , lactate dehydrogenase; 6 , citrate synthase; 7 , phosphotransacetylase; 8 , acetate kinase.

2. The mechanism which couples oxidation of intermediates to energy generation may 'slip' so that excess carbon is dissipated as $\mathrm{CO}_{2}$ and excess energy is liberated as heat (Neijssel \& Tempest, 1979; Stouthamer, 1979; Roels, 1980; Tempest \& Neijssel, 1984).

3. The surplus capacity of the central pathways can be dissipated by excretion of low molecular mass compounds into the extracellular water space (Demain, 1972; Meyer et al., 1984; Holms, 1986).

Whichever mechanism is employed, the end result is the same - the fluxes through the central pathways to provision of precursors and energy are balanced to the fluxes of these components for growth. This paper defines an example of the third mechanism in which flux of carbon into the central pathways exceeds the requirements for growth and is balanced by the excretion of acetate. Among the compounds tested in this study, pyruvate sustains the largest flux to acetate excretion and is central to the problem of acetate excretion as, irrespective of the nature of the primary carbon source, all carbon diverted to acetate is derived from it as illustrated in Fig. 1. The purpose of the work reported here was to define this system and devise means by which the wasteful flux of pyruvate feedstock could be prevented.

\section{METHODS}

Chemicals and enzymes. Fluoroacetic acid, sodium salt, was obtained from Aldrich. All other chemicals were as previously described (Clark \& Holms, 1976; El-Mansi et al., 1986), or were of AnalaR grade. With the exception of the acetate-assay enzymes, i.e. acetyl-CoA synthetase, citrate synthase and malate dehydrogenase, which were obtained from Boehringer, all enzymes were from Sigma. 
Bacterial strains. Escherichia coli ATCC 15224 (ML308) and its fluoroacetate-resistant derivative were used. The parent strain, apart from being constitutive for the lac operon $\left(i^{-} z^{+} y^{+} a^{+}\right)$, was considered to be a wild-type. Inocula were grown on the relevant substrate for 18 generations as previously described by Holms \& Bennett (1971). Fluoroacetate-resistant strains were selected for their ability to grow on pyruvate (25 mM) agar minimal medium containing fluoroacetate (10 mM) as previously described by Brown et al. (1977).

Growth conditions. (a) Batch culture. The parent as well as its fluoroacetate-resistant derivative were grown in batch cultures $\left(800 \mathrm{ml}\right.$ ) aerobically at $37^{\circ} \mathrm{C}$ (Harvey et al., 1968) in a simple defined medium (Hamilton \& Holms, 1970) supplemented with the appropriate carbon source.

(b) Continuous culture. Media and growth conditions were as previously described by Clark \& Holms (1976). While the 2 litre bioreactor fermenter from Life Science Laboratories was used for most of the experiments, an apparatus (Clark \& Holms, 1976) based on the design of Baker (1968), was also used in the early part of this work. Growth at a maximum specific growth rate $\left(\mu_{\max }\right)$ was achieved by manually regulating the flow rate, frequently measuring optical density and adjusting the dilution rate so that a constant bacterial density was maintained. Under these conditions excess primary carbon source was always present in the effluent (Fig. 4). Carbon limitation however, was created by gradually decreasing the dilution rate and allowing the chemostat to reach a steady state prior to sampling. Bacterial growth was followed by measuring the optical density at $420 \mathrm{~nm}$ in a single-beam flow-cell Pye Unicam SP30 spectrophotometer. An optical density of 1.0 corresponds to $196 \mu \mathrm{g} \mathrm{dry} \mathrm{wt}$ cells $\mathrm{ml}^{-1}$.

Detection and measurements of the primary carbon source and the excreted acetate. Concentration of the primary carbon source and acetate were determined in the culture filtrate $(0.22 \mu \mathrm{m}$ Millipore filter) either enzymically as previously described (Bücher et al., 1963; Bergmeyer \& Möllering, 1974) or using HPLC analysis by reference to standards, the concentrations of which were first established enzymically.

HPLC. This was done at room temperature on Aminex HPX-87H organic acid columns (Bio-Rad). Samples $(25-100 \mu \mathrm{l})$ were applied to the column and eluted using $25 \mathrm{mM}-\mathrm{H}_{2} \mathrm{SO}_{4}$ at a flow rate of $1 \mathrm{ml} \mathrm{min} \mathrm{m}^{-1}$ and monitored at $215 \mathrm{~nm}$. The identities of the eluted peaks were verified using authentic materials.

\section{RESULTS}

\section{Excretion of metabolites during growth in batch cultures}

During growth of $E$. coli ML308 on pyruvate, a large fraction of the primary carbon source is excreted in the form of acetate (Fig. $2 b$ ). Once pyruvate is exhausted the organism utilizes the excreted acetate to support a second phase of growth (Fig. $2 a$ ), which in comparison with the first phase is both poor and slow. Adaptation to acetate following the exhaustion of pyruvate was concomitant with a threefold inactivation of isocitrate dehydrogenase (EC 1.1.1.42) and the derepression of the glyoxylate bypass enzyme isocitrate lyase (EC 4.1.3.1), which is consistent with other reports from this laboratory (Bennett \& Holms, 1975; El-Mansi et al., 1986). In the first phase, lactate was excreted $\left[0.52 \pm 0.08 \mathrm{mmol}(\mathrm{g} \text { dry } w \mathrm{t})^{-1} ; n=4\right]$, but this was quantitatively insignificant when compared with acetate excretion $[24 \pm 0.75 \mathrm{mmol}$ (g dry $\left.w t)^{-1} ; n=4\right]$. No lactate was detected in the second phase of growth.

\section{Effect of 3-bromopyruvate on acetate excretion}

During growth in batch culture $\left(\mu=0.72 \pm 0.09 \mathrm{~h}^{-1} ; n=8\right)$, partition of carbon flux at the junction of pyruvate (Fig. 1) is resolved in favour of pyruvate dehydrogenase (PDH; EC 1.2.4.1) as evidenced by the excretion of acetate. It follows that restriction of carbon flux through PDH is one way to bring about the diminution of flux to acetate excretion. Among those compounds which act as active-site-directed inhibitors of PDH, 3-bromopyruvate is the most effective and its mode of action is well-documented (Bisswagner, 1981; Lowe \& Perham, 1984). During growth on pyruvate $(7.5 \mathrm{mM})$ bromopyruvate $(50 \mu \mathrm{M})$ prevented flux to acetate excretion (but not lactate) and improved the efficiency of carbon conversion to biomass from 18.9 to $23.3 \mathrm{~g}$ dry wt (mol substrate) ${ }^{-1}$ (some $23 \%$ ) but this was at the expense of growth rate $\left(\mu=0.30 \pm 0.08 \mathrm{~h}^{-1} ; n=3\right)$ (Fig. $\left.2 a\right)$.

\section{Excretion of carbon during growth of fluoroacetate-resistant strains of E. coli ML308 on pyruvate}

Fluoroacetate-resistant derivatives of E. coli (Brown et al., 1977; Guest, 1979; Levine et al., 1980), Salmonella typhimurium (Levine et al., 1980) and other organisms, e.g. Azotobacter vinelandii (McKenney \& Melton, 1986) and Aerobacter aerogenes (Brown et al., 1972) are unable 

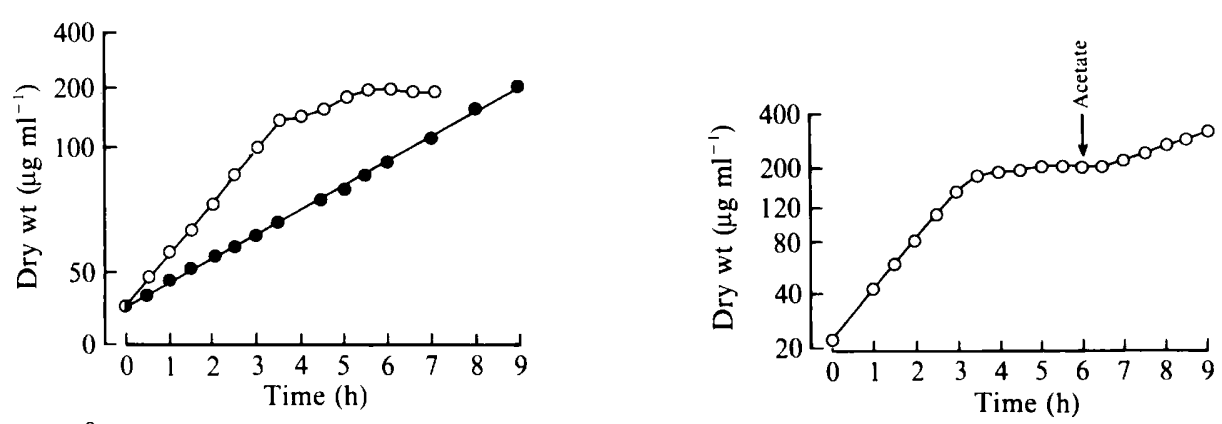

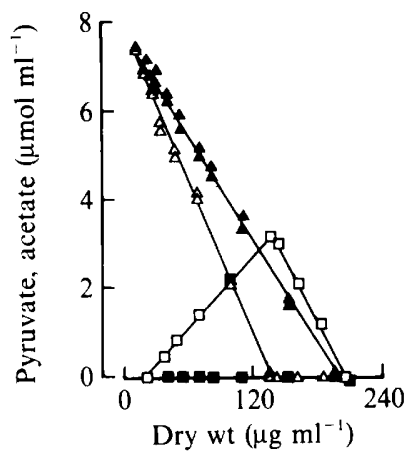

Fig. 2

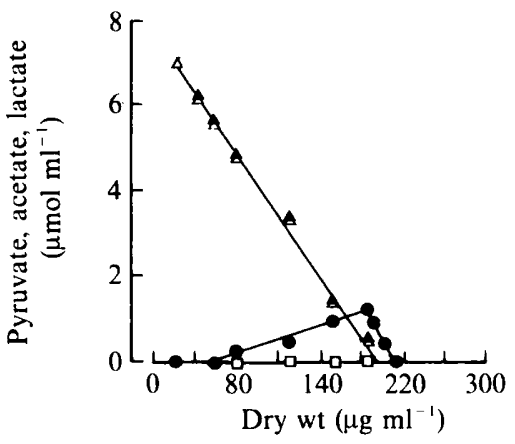

Fig. 3

Fig. 2. Effect of 3-bromopyruvate on growth, acetate excretion and substrate utilization during growth of $E$. coli ML308 on pyruvate $\left(7.5 \mathrm{mM}\right.$ ) minimal medium in batch culture at $37^{\circ} \mathrm{C}$. 3-Bromopyruvate was added to the culture just before inoculation and growth $(O, O)$ was followed by measuring the optical density at $420 \mathrm{~nm}$. Pyruvate $(\Delta, \triangle)$ was assayed in the culture filtrates $(0 \cdot 22 \mu \mathrm{m}$ Millipore filter $)$ enzymically while acetate $(\square, \square)$ was assayed either enzymically or by HPLC as described in the text. Closed symbols, values obtained in the presence of $50 \mu \mathrm{M}$-bromopyruvate; open symbols, no bromopyruvate present.

Fig. 3. Acetate $(\square)$ and lactate $(\bigcirc)$ excretion and pyruvate utilization $(\Delta, \Delta)$ during growth $(O)$ of the fluoroacetate-resistant strain $E$. coli ML308/EM39 on pyruvate minimal medium in batch culture. Growth conditions and measurements of acetate and pyruvate were as described in the legend to Fig. 2. Lactate was identified by HPLC and quantified enzymically using lactate dehydrogenase.

to convert acetate to acetyl-CoA by acetate kinase (ATP:acetate phosphotransferase; EC 2.7.2.1) and phosphotransacetylase (acetyl-CoA:orthophosphate acetyltransferase; EC 2.3.1.8). Fluoroacetate-resistant mutants of the organism under investigation appeared at a frequency of 9.13-9.68 $\times 10^{-7}$. The three selected for further study all gave similar results and did not excrete acetate (Fig. 3). The growth rate $\left(\mu=0.63 \mathrm{~h}^{-1}\right)$ was less than the parent $\left(\mu=0.72 \mathrm{~h}^{-1}\right)$ but pyruvate uptake was still excessive and carbon flow was balanced by the excretion of lactate $\left[5.57 \pm 0.34 \mathrm{mmol}(\mathrm{g} \mathrm{dry} \mathrm{wt})^{-1} ; n=3\right]$ which was co-utilized with pyruvate when the concentration of the latter in the medium fell below about $1.2 \mathrm{~mm}$. Rather unexpectedly, acetate added after exhaustion of pyruvate sustained growth, albeit rather slowly $\left(\mu=0 \cdot 21 \mathrm{~h}^{-1}\right)$.

\section{Excretion of acetate during growth in continuous cultures}

(a) Flux to acetate excretion during growth in a turbidostat. In agreement with the results obtained from batch culture experiments, some $28 \%$ of the total carbon was excreted as acetate during growth in a turbidostat $\left(\mu=0.729+0.02 \mathrm{~h}^{-1} ; n=3\right)$. Under these circumstances, it was also established that flux to acetate excretion was proportional to biomass and carbon supply (Fig. 4).

(b) Flux to acetate excretion during growth in a chemostat. Restriction of carbon supply in a chemostat diminished flux to acetate excretion (Fig. 5). At a dilution rate of $0.35 \mathrm{~h}^{-1}$ or less no 


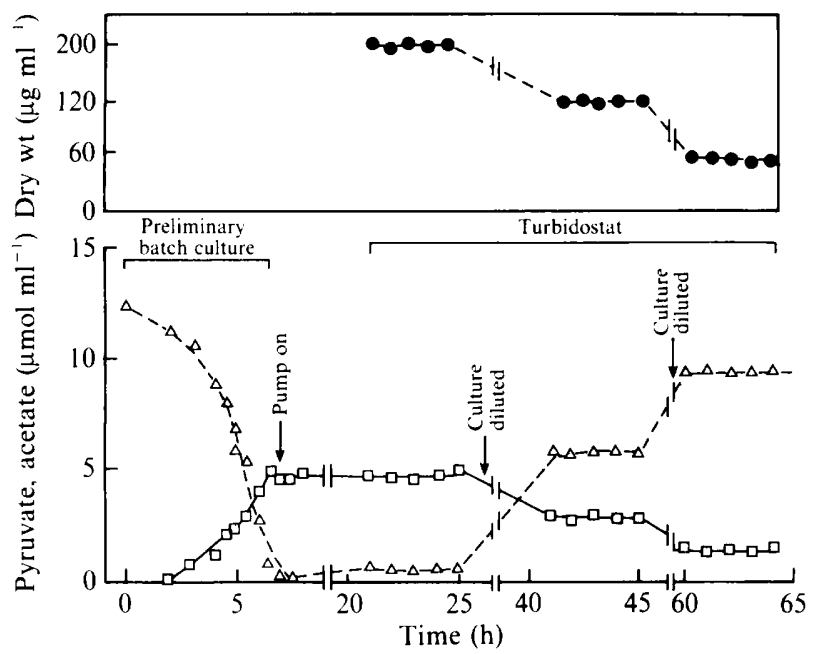

Fig. 4. Acetate excretion $(\square)$ and pyruvate utilization $(\triangle)$ during growth (O) of E. coli ML308 in a turbidostat at three different concentrations of biomass.

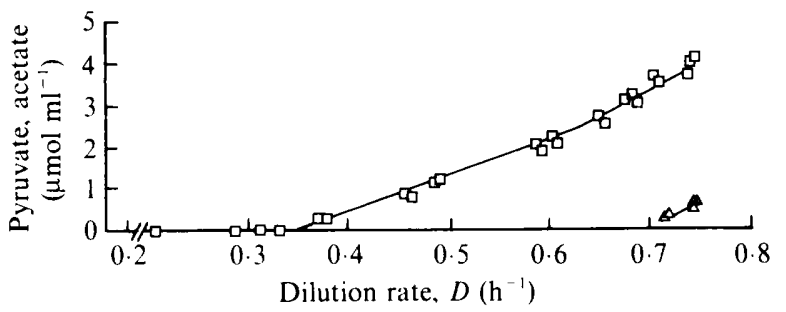

Fig. 5. Effect of dilution rate on acetate excretion during growth of $E$. coli ML308 on pyruvate in a continuous culture at $37^{\circ} \mathrm{C}$. Acetate $(\square)$ and pyruvate $(\triangle)$ were assayed as described in the text.

flux to acetate excretion was sustained thus permitting perfect balance between carbon input on one hand and the output to biosynthesis and energy generation on the other. This, in turn, is reflected in the efficiency of carbon conversion to biomass.

\section{DISCUSSION}

When E. coli ML308 grows on pyruvate the uptake of carbon source exceeds the capacity of the organism to generate biomass and the system is balanced by excretion of acetate (Fig. 6). This strategy is advantageous to the organism as flux of acetyl-CoA through phosphotransacetylase allows the pool of free reduced CoA to be regenerated. Moreover, flux through acetate kinase yields $1 \mathrm{~mol}$ of ATP for each mol of acetate excreted (Rose et al., 1954; Brown et al., 1977; Thauer et al., 1977). It is also possible that passage of acetate across the membrane helps to maintain the protonmotive force (Thauer et al., 1977; Ten Brink \& Konings, 1980).

In continuous culture, turbidostats (Fig. 4) give essentially the same results as batch cultures (Fig. 2). When the rate of pyruvate uptake is restricted in chemostats, to less than about 17 mmol (g dry wt) $)^{-1} \mathrm{~h}^{-1}\left(\mu=0.35 \mathrm{~h}^{-1}\right)$ no acetate is excreted (Fig. 7). Pyruvate provides direct entry to the central pathways (Figs 1 and 6) and sustains a relatively high growth rate $\left(\mu=0.72 \mathrm{~h}^{-1}\right)$, which in turn is a reflection of a rapid turnover of metabolic intermediates to satisfy biosynthesis. 


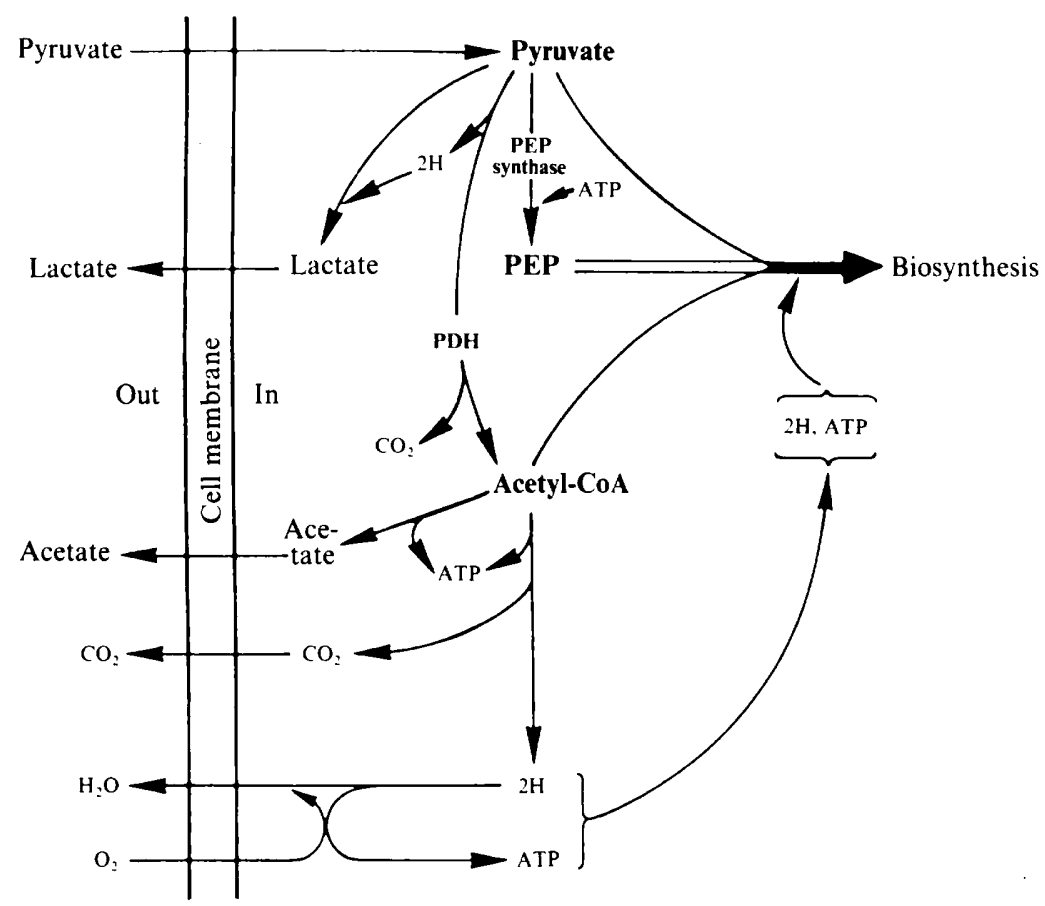

Fig. 6. Carbon fluxes to biosynthesis, acetate and lactate excretion, and energy balance during growth of $E$. coli on pyruvate.

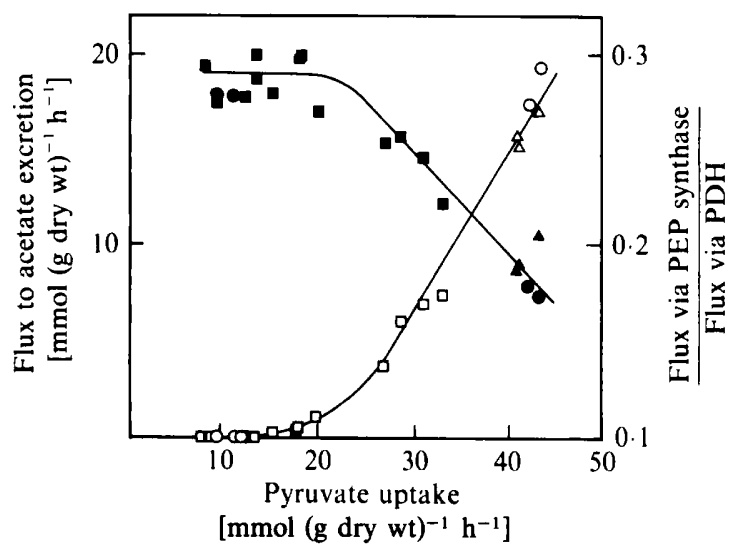

Fig. 7. Effect of the rate of pyruvate uptake on the flux of carbon to acetate excretion $(O$, batch cultures; $\triangle$, turbidostat; $\square$, chemostat) and the flux through PEP synthase relative to PDH (Q, batch culture; $\boldsymbol{\Lambda}$, turbidostat; $\boldsymbol{D}$, chemostat) during growth of $E$. coli ML308 on pyruvate in batch and continuous cultures at $37^{\circ} \mathrm{C}$ under aerobic conditions.

In all the different types of culture used in this work, the input of pyruvate and the output of biomass and excreted carbon compounds was measured. The monomeric composition of the biomass (Morowitz, 1968) was used to derive the outputs of precursors required to generate that biomass and to relate, in quantitative terms, the flow of carbon from input to outputs in terms of throughputs (all three being expressed in mmol required to generate $1 \mathrm{~g}$ dry wt of new biomass). These throughputs can then be converted to true fluxes [ $\mathrm{mmol}(\mathrm{g} \text { dry wt) })^{-1} \mathrm{~h}^{-1}$ ] if the growth rate ( $\mu$ in terms of $h^{-1}$ ) is also measured. Both throughputs and fluxes are then an exact description of 
Table 1. Effect of growth rate $(\mu)$ on the partition of carbon fluxes $(\%)$ at the junctions of pyruvate and acetyl-CoA during growth of E. coli ML308 on pyruvate in continuous cultures

Fluxes [mmol ( $\mathrm{g}$ dry wt $\left.)^{-1} \mathrm{~h}^{-1}\right]$ of pyruvate were taken as $100 \%$ and the relative fluxes to acetyl-CoA, PEP and biosynthesis were calculated. The relative distribution of the acetyl-CoA generated among the routes for biosynthesis, energy generation $\left(\mathrm{CO}_{2}\right)$ and acetate excretion was also determined. The data shown below for the turbidostat and the chemostats were derived from their corresponding flux diagrams, each of which was based on the mean of at least four independent values.

\begin{tabular}{|c|c|c|c|c|c|c|c|}
\hline & & & yruva & & & yl-CoA & \\
\hline & $\mu$ & Acetyl-CoA & PEP & Biosynthesis & Biosynthesis & Acetate & $\mathrm{CO}_{2}$ \\
\hline Turbidostat & 0.729 & $81 \cdot 4$ & $14 \cdot 2$ & 3.9 & $4 \cdot 5$ & $39 \cdot 2$ & $37 \cdot 8$ \\
\hline & 0.681 & $77 \cdot 8$ & $17 \cdot 4$ & $4 \cdot 7$ & $5 \cdot 4$ & $24 \cdot 4$ & $47 \cdot 9$ \\
\hline & 0.606 & $75 \cdot 8$ & $19 \cdot 0$ & $5 \cdot 2$ & 6.0 & $14 \cdot 0$ & 55.9 \\
\hline Chemostat & 0.471 & $74 \cdot 7$ & $19 \cdot 9$ & $5 \cdot 5$ & $6 \cdot 2$ & $6 \cdot 5$ & $62 \cdot 0$ \\
\hline & 0.372 & $74 \cdot 0$ & $20 \cdot 4$ & 5.6 & $6 \cdot 4$ & 1.8 & 65.8 \\
\hline & 0.303 & $74 \cdot 1$ & $20 \cdot 3$ & $5 \cdot 6$ & $6 \cdot 8$ & 0.0 & $67 \cdot 3$ \\
\hline
\end{tabular}

the metabolic events occurring in the central metabolic pathways and the only assumption on which they depend is that the metabolic routes assumed to be used actually function in the biomass (Holms, 1986). The junctions at pyruvate and acetyl-CoA are the most significant for adjustment of throughput relative to the outputs of precursors and excreted products. The relative distribution of fluxes in continuous cultures at these junctions is given in Table 1 . As the input of pyruvate is diminished the fluxes from pyruvate to biosynthesis (both directly and via PEP synthase) are enhanced. Similarly flux of carbon from acetyl-CoA to biosynthesis and aerobic energy generation (i.e. $\mathrm{CO}_{2}$ production in the $\mathrm{Krebs}$ cycle) is enhanced while flux to acetate excretion is diminished. A good index of these relative changes is a comparison of the fluxes through PEP synthase, which is exclusively dedicated to provision of precursors for biosynthesis, and PDH, which sustains fluxes to biosynthesis (acetyl-CoA), excretion (acetate) and energy generation, by $\mathrm{CO}_{2}$ production and oxidative phosphorylation (both by itself and subsequently in the Krebs cycle). The ratio of fluxes of PEP synthase to PDH increases as acetate excretion is diminished relative to pyruvate uptake in continuous and various batch cultures (Fig. 7). When pyruvate input is balanced to biosynthesis and acetate excretion is eliminated the relative fluxes through PEP synthase and PDH remain constant even when pyruvate input is further restricted (Fig. 7). It follows that excessive pyruvate input is dissipated primarily by flux through PDH en route to acetate excretion.

Inclusion of 3-bromopyruvate prevents flux to acetate excretion (Fig. $2 b$ ) by virtue of its ability to diminish flux to acetyl-CoA both directly, by diminishing flux through PDH (Lowe \& Perham, 1984) and indirectly, by diminishing flux of pyruvate into the cell (Lang et al., 1987). The ability of bromopyruvate to restrict the uptake of pyruvate explains the absence of lactate excretion under these circumstances.

Assimilation of fluoroacetate gives rise to fluoroacetyl-CoA which in turn is converted, by condensation with oxaloacetate, to fluorocitrate. Fluorocitrate is a potent inhibitor of the Krebs cycle enzyme aconitase (EC 4.2.1.3) (Mager et al., 1955; Marcus \& Elliott, 1959). It follows that mutants resistant to fluoroacetate must be deficient in one or both of the enzymes involved in the conversion of fluoroacetate to fluoroacetyl-CoA, namely acetate kinase and phosphotransacetylase. The activities of these enzymes are reversible and are therefore used for acetate excretion. It is hardly surprising, therefore, that such mutants are impaired in their ability to excrete acetate (Fig. $3 b$ ). However, under these circumstances excessive flux of pyruvate to the central pathways is balanced by diversion of carbon flux to lactate excretion (Fig. $3 b$ ). This strategy is forced upon the mutant because it cannot convert acetyl-CoA to acetate for excretion. However, pyruvate-grown cultures of such mutants can still metabolize acetate via the activity of acetyl-CoA synthetase (acetate :CoA ligase, AMP forming; EC 6.2.1.1) (Overath et al., 1969), an irreversible enzyme that cannot therefore be used for acetate excretion. The possibility 
that another enzyme is involved under these circumstances for the activation of acetate to acetylCoA cannot be ruled out.

\section{CONCLUSIONS}

Precursors for biosynthesis are removed from the central metabolic pathways at rates related to the rate of generation of new biomass $(\mu)$. The rate of pyruvate uptake exceeds these requirements and the surplus carbon is removed from the central metabolic pathways and excreted as acetate. As long as the mechanism for acetate excretion is available (phosphotransacetylase and acetate kinase) the only way in which acetate excretion can be avoided is to diminish the rate of flux of pyruvate into the cell. This can be achieved by inhibition of pyruvate uptake and its conversion to acetyl-CoA by 3-bromopyruvate or by restricting the rate of pyruvate supply in a chemostat. In both cases, however different in nature, flux to acetate excretion is diminished thus permitting a more efficient use of acetyl-CoA for energy generation. Flux to acetate excretion was totally prevented when the growth rate $(\mu)$ was reduced to $0.35 \mathrm{~h}^{-1}$ or less. Fluoroacetate-resistant mutants, impaired in their ability to excrete acetate, dissipate excessive flux of pyruvate by the excretion of lactate.

When flux of carbon source into the central pathways exactly matches the biosynthetic demands there is no flux to acetate excretion, e.g. during growth on glycerol (Holms, 1986). It seems probable, therefore, that metabolites of the central pathways are excreted when flux of carbon into the cell exceeds the demands for biosynthesis and energy generation. Furthermore, flux to acetate excretion is not a consequence of oxygen deficiency, but rather the saturation of the electron transport chain.

This work was done under contract to the Eastern Electricity Board. The encouragement and assistance of $\mathrm{Dr} J$. Curran is gratefully acknowledged.

We wish to pay tribute to the late Professor R. M. S. Smellie for his encouragement and constant interest.

\section{REFERENCES}

BAKER, K. (1968). Low cost continuous culture apparatus. Laboratory Practice 17, 817-824.

BenNeTT, P. M. \& Holms, W. H. (1975). Reversible inactivation of the isocitrate dehydrogenase of Escherichia coli ML308 during growth on acetate. Journal of General Microbiology 87, 37-51.

Bergmeyer, H. U. \& Möllering, H. (1974). Acetate determination with preceding indicator reaction. In Methods of Enzymatic Analysis, 2nd edn, vol. 3, pp. 1520-1527. Edited by H. U. Bergmeyer. New York \& London: Academic Press.

Bisswagner, H. (1981). Substrate specificity of the pyruvate dehydrogenase complex from Escherichia coli. Journal of Biological Chemistry 256, 815-822.

Brown, T. D. K., Pereira, C. R. S. \& Stormer, F. C. (1972). Studies of the acetate kinase-phosphotransacetylase and the butanediol-forming systems in Aerobacter aerogenes. Journal of Bacteriology 112, 1106-1111.

Brown, T. D. K., Jones-Mortimer, M. C. \& KORNBERG, H. L. (1977). The enzymic interconversion of acetate and acetyl-coenzyme A in Escherichia coli. Journal of General Microbiology 102, 327-336.

BÜChER, T., CZOK, R., LAMPRECHT, W. \& LATZKo, E. (1963). Pyruvate. In Methods of Enzymatic Analysis, pp. 253-259. Edited by H. U. Bergmeyer. London \& New York: Academic Press.

Clark, B. \& Holms, W. H. (1976). Control of the sequential utilization of glucose and fructose of Escherichia coli. Journal of General Microbiology 95, 191-201.

Dawes, E. A. \& Senior, P. J. (1973). The role and regulation of energy reserve polymers in microorganisms. Advanced Microbial Physiology 10, 135-266.

DAWES, E. A. \& LARGE, P. J. (1982). In Biochemistry of Bacterial Growth, pp. 125-183. Edited by J. Mandelstam, K. McQuillan \& I. Dawes. Oxford: Blackwell Scientific Publications.

Demain, A. L. (1972). Cellular and environmental factors affecting the synthesis and excretion of metabolites. Journal of Applied Chemical Biotechnology 22, 345-362.

Dietzler, D. N., Leckie, M. P., Sternheim, W. L., Ungar, J. M., Crimmins, D. L. \& Lewis, J. W. (1979). Regulation of glucose utilization in Escherichia coli during maintenance of the energy charge. Journal of Biological Chemistry 254, 8276-8287.

El-Mansi, E. M. T., Nimmo, H. G. \& Holms, W. H. (1986). Pyruvate metabolism and the phosphorylation state of isocitrate dehydrogenase in Escherichia coli. Journal of General Microbiology 132, 797-806.

GUEST, J. R. (1979). Anaerobic growth of Escherichia coli K12 with fumarate as terminal electron acceptor: genetic studies with menaquinone and fluoroacetate-resistant mutants. Journal of General Microbiology 115, 259-271. 
Hamilton, I. D. \& Holms, W. H. (1970). Measurement of respiration of micro-organisms during batch culture. Laboratory Practice 19, 797-798.

Harvey, N. L., Fewson, C. A. \& Holms, W. H. (1968). Apparatus for batch culture of micro-organisms. Laboratory Practice 17, 1134-1136.

Holms, W. H. (1986). The central metabolic pathways of Escherichia coli: relationship between flux and control at a branch point, efficiency of conversion to biomass, and excretion of acetate. Current Topics in Cellular Regulation 28, 59-105.

Holms, W. H. (1987). Control of flux through the citric acid cycle and the glyoxylate bypass in Escherichia coli. Biochemical Society Symposia 54, 17-31.

Holms, W. H. \& BenNeTt, P. M. (1971). Regulation of isocitrate dehydrogenase activity in Escherichia coli on adaptation to acetate. Journal of General Microbiology 65, 57-68.

LANG, V. J., Leystra-LANTZ, C. \& CoOK, R. A. (1987). Characterization of the specific pyruvate transport system in Escherichia coli K-12. Journal of Bacterio$\log y 169,380-385$.

LeVINE, S. M., ARDEShIR, F. \& AMEs, G. F.-L. (1980). Isolation and characterization of acetate kinase and phosphotransacetylase mutants of Escherichia coli and Salmonella typhimurium. Journal of Bacteriology 143, 1081-1085.

Lowe, P. N. \& Perham, R. N. (1984). Bromopyruvate as an active-site-directed inhibitor of the pyruvate dehydrogenase multienzyme complex from Escherichia coli. Biochemistry 23, 91-97.

MCKenNEY, D. \& MELTON, T. (1986). Isolation and characterization of ack and pta mutations in Azotobacter vinelandii affecting acetate-glucose diauxie. Journal of Bacteriology 165, 6-12.

Mager, J., Goldblum-Sinai, J. \& Blank, I. (1955). Effect of fluoroacetic acid and allied fluoroanalogues on growth of Escherichia coli. Journal of Bacteriology 70, 320-325.

Marcus, A. \& Elliott, W. B. (1959). Enzymatic reactions of fluoroacetyl phosphate. Journal of Biological Chemistry 234, 1011-1014.
Meyer, H. P., Leist, C. \& Fiechter, A. (1984). Acetate formation in continuous culture of Escherichia coli $\mathrm{K} 12 \mathrm{D} 1$ on defined and complex media. Journal of Biotechnology 1, 355-358.

Morowitz, H. J. (1968). Energy Flow in Biology: Biological Organization as a Problem in Thermal Physics, p. 179. New York: Academic Press.

NeidhardT, F. C., IngrahaM, J. L., Low, K. B., Magasanik, B., Schaechter, M. \& UMbarger, H. E. (1987). Escherichia coli and Salmonella typhimurium: Cellular and Molecular Biology, vol. 1, p. 806. Washington, DC: American Society for Microbiology.

Neijssel, O. M. \& Tempest, D. W. (1979). The physiology of metabolite overproduction. Symposia of the Society for General Microbiology 29, 53-82.

Overath, P., Paul, G. \& Schairer, H. U. (1969). Fatty acid degradation in Escherichia coli: an inducible acyl-CoA synthetase, the mapping of oldmutations, and the isolation of regulatory mutants. European Journal of Biochemistry 7, 559-574.

Roels, J. A. (1980). Application of macroscopic principle to microbial metabolism. Biotechnology and Bioengineering 22, 2457-2514.

Rose, I. A., Grunberg-Manago, M., Korey, S. \& OCTTOA, S. (1954). Enzymatic phosphorylation of acetate. Journal of Biological Chemistry 211, 737-756.

Stouthamer, A. H. (1979). In Microbial Biochemistry, pp. 1-47. Edited by J. R. Quayle. Baltimore: University Park Press.

Tempest, D. W. \& NeIJSsel, O. M. (1984). The status of ATP and maintenance energy as biologically interpretable phenomena. Annual Review of Microbiology 38, 459-486.

Ten Brink, B. \& Konings, W. N. (1980). Generation of an electrochemical proton gradient by lactate efflux in membrane vesicles of Escherichia coli. European Journal of Biochemistry 111, 59-66.

Thauer, R., JungermanN, K. \& DeCKER, K. (1977). Energy conservation in chemotrophic anaerobic bacteria. Bacteriological Reviews 41, 100-180. 